\title{
Mathematical modelling for the comparison of plate and intramedullary osteosynthesis stability in intraarticular distal radius fractures
}

\author{
Vlcek $\mathrm{M}^{1}$, Landor $\mathrm{I}^{2}$, Horak $\mathrm{Z}^{3}$, Musil $\mathrm{V}^{4}$, Sosna $\mathrm{A}^{2}$, Jonas $\mathrm{D}^{2}$ \\ Second Medical Faculty, Charles University in Prague, Teaching Hospital Motol, 1st Clinic of Orthopaedic Surgery, \\ Department of Traumatology, Prague, Czech Republic. dr.martinvlcek@gmail.com
}

\begin{abstract}
Background: A comparison between plate and intramedullary osteosynthesis techniques in terms of the biomechanical stability of distal radius fracture fixation.

Methods: Mathematical modelling was used to simulate distal radius fractures, type $\mathrm{C} 1$ and type $\mathrm{C} 2$, and to compare the stability of osteosynthesis in both techniques under several wrist joint load conditions: axial compressive loading of the radius, bending loads in volar and dorsal flexion and radial and ulnar deviation, and axial torsional loading of the forearm. Results: For both type C1 and type C2 fractures, the stability of intramedullary osteosynthesis was comparable with plate osteosynthesis in dorsal flexion. Plate osteosynthesis proved to be more stable only in ulnar deviation. The intramedullary X-screw technique provided more stability when loads were applied in volar flexion, radial deviation, pronation and supination, and in axial loading of the radius.

Conclusions: The result of mathematical simulations was comparable for both types of fracture. It showed a higher stability of intramedullary osteosynthesis. Although when lower force intensity was applied, both techniques were comparable (Fig. 8, Ref. 16). Text in PDF www.elis.sk.

Keywords: mathematical modelling, distal radius fracture, locking plate osteosynthesis, intramedullary osteosynthesis, stability.
\end{abstract}

The current preferred method for the surgical treatment of distal radius fractures is volar locking plate osteosynthesis. Various designs of angle-stable plates pre-contoured to fit the distal radius are available and are very similar in their properties and outcomes (1-4). Although this method is versatile for a broad range of indications, attempts to use less invasive methods for simple fractures shouldn't be ignored. It includes development of various implants for intramedullary osteosynthesis of distal radius fractures (such as Targon DR, Micronail or X-screw). Promising results with few complications have been reported by Dutch researches in extraarticular and partial intra-articular fractures treated by intramedul-

${ }^{1}$ Second Medical Faculty, Charles University in Prague, Teaching Hospital Motol, 1st Clinic of Orthopaedic Surgery, Department of Traumatology, Prague, Czech Republic, ${ }^{2}$ First Faculty of Medicine, Charles University in Prague, Teaching Hospital Motol, 1st Clinic of Orthopaedic, Surgery, Prague, Czech Republic, ${ }^{3}$ Czech Technical University in Prague, Faculty of Mechanical Engineering, Department of Mechanics, Biomechanics and Mechatronics, Laboratory of Biomechanics, Prague, Czech Republic, ${ }^{4}$ Third Faculty of Medicine, Charles University in Prague, Centre of Scientific Information, Prague, Czech Republic

Address for correspondence: I. Landor, MD, PhD, First Faculty of Medicine, Charles University in Prague, Teaching Hospital Motol, 1st Clinic of Orthopaedic Surgery, Department of Traumatology, V Uvalu 84, CZ-150 06 Prague 5, Czech Republic.

Phone: +420.224433901, Fax: +420.224438623

Acknowledgement: This study was supported by the Czech Ministry of Education, Youth and Sports project: "Transdisciplinary research in Biomedical Engineering II", No. MSM 6840770012.

Supported by the research project of the Charles University in Prague P38

- Biological aspects of the investigation of human motricity lary fixation. In this study, the Micronail fixation system provided a good stability for the wrist. All fractures healed without major loss of alignment and outcomes were comparable with those of plate osteosynthesis (5).

German authors achieved positive results with the screw-platehybrid Targon DR in surgical treatment of type A2, A3 and B1 fractures (6). However, intramedullary osteosynthesis is unsuitable for type B2 and B3 fractures since the fracture line is running in the sagittal plane (7). The use of this method in type $\mathrm{C}$ fractures is still debatable.

In intramedullary osteosynthesis, all implants are inserted from the radial approach through the radial styloid process and this requires the presence of a sufficiently large fragment. Distal epiphysis fragments located medially to this large fragment is in this technique secured only with screws or wires that are not anglestable with the implant. Therefore, osteosynthesis can be stable enough on the condition that the bone is not comminuted. In view of this, only type $\mathrm{C} 1$ and $\mathrm{C} 2$ fractures can be treated by this method.

The aim of our experimental study was to compare the plate osteosynthesis and intramedullary $\mathrm{X}$-screw osteosynthesis, in terms of biomechanical stability of fixation, in distal radius type $\mathrm{C} 1$ and $\mathrm{C} 2$ fractures.

\section{Material and methods}

Mathematical modelling for the comparison of a locking plate and an intramedullary osteosynthesis was based on the anatomical angle-stable T-plate (Medin a.s, Nové Město na Moravě, Czech Re- 


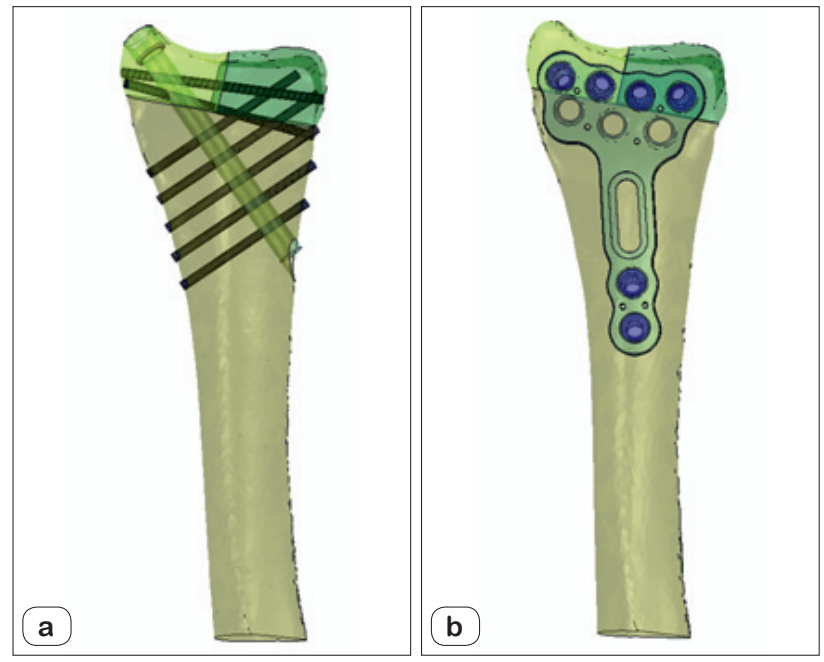

Fig. 1. Mathematical simulations of type $\mathbf{C} 2$ distal radius fractures fixed using: a) intramedullary osteosynthesis, b) plate osteosynthesis.

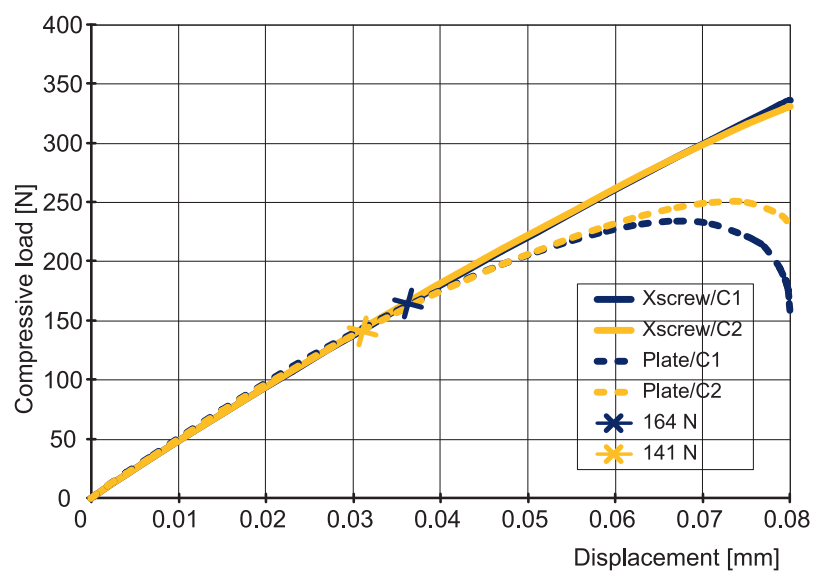

Fig. 2. Relationship between a compressive force and a magnitude of displacement under axial loading of the radius.

public) and the intramedullary X-screw system (Zimmer, Inc., Warsaw, Indiana, USA). The former was selected because its technical data were available from the time of its construction, and the latter because its previous use showed the most problematic clinical results. The stability of osteosynthesis with these implants was evaluated in type $\mathrm{C} 1$ and $\mathrm{C} 2$ distal radius fractures whose models consisted of three and six bone fragments, respectively. Totally, four mathematical models were created (Fig 1). Deformation and stress responses of the implants, the bone fragments to model loading of the distal radius region were evaluated by the finite element method (FEM).

The mathematical model of an angle-stable plate was provided by the Medin company in the *SAT format, an electronic version $3 \mathrm{D}$ geometric models. This model was imported into the ABAQUS 6.9 software (Simulia, Providence, Rhode Island, USA), which allows for finite element modelling of loads imposed on either parts of or the whole of a system by external forces. The simulation results presented information on the distribution and magnitude of both stress and deformation in the part considered, i.e., a fracture fixed with an osteosynthetic implant.

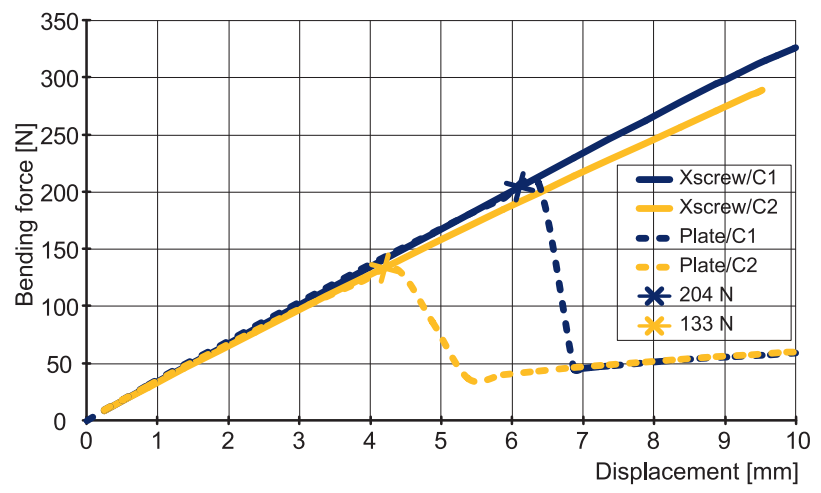

Fig. 3. Relationship between a bending force and a magnitude of displacement in volar flexion of the wrist.

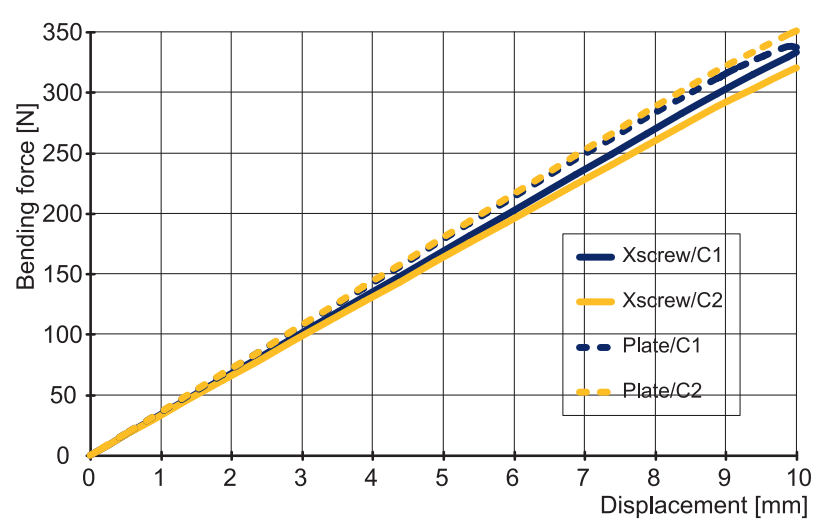

Fig. 4. Relationship between a bending force and a magnitude of displacement in dorsal flexion of the wrist.

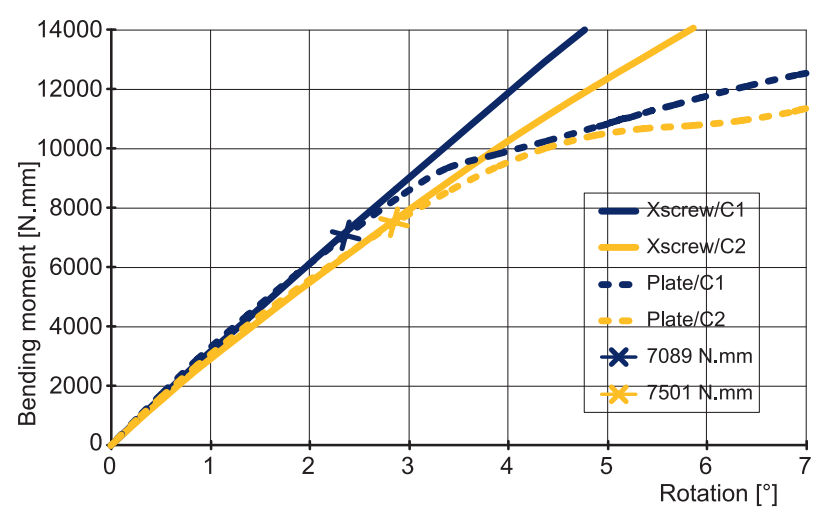

Fig. 5. Relationship between a bending force and a magnitude of displacement in radial deviation of the wrist.

The geometric model of an intramedullary X-screw system was based on a physical screw manufactured by Rhinoceros 4.0 and Commercial software for three-dimensional NURBS modelling (McNeel, Seattle, Washington, U.S.A.). Both the screw and the plate models were imported in a *SAT format, into ABAQUS 6.9 software. To simplify the calculations for the implant parts containing screw threads, all these parts were substituted with the smooth surface of a cylinder running through half of the width of screw threads. This method is common and widely used in mechanical 


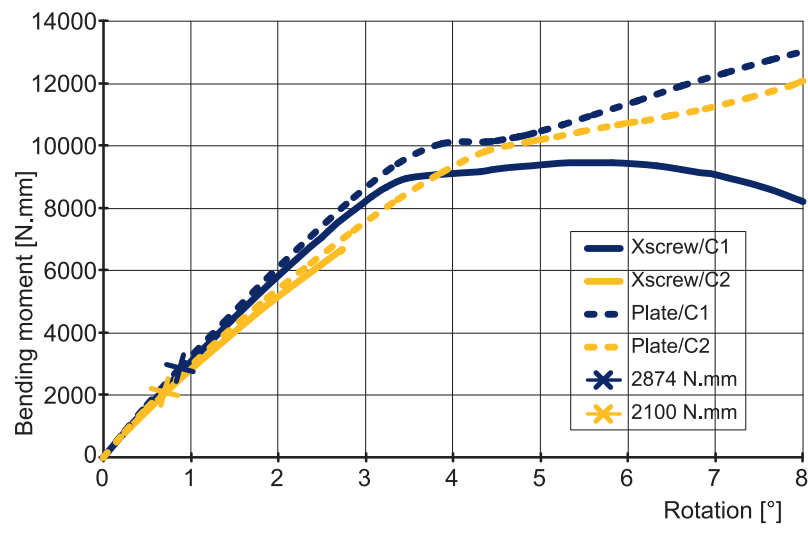

Fig. 6. Relationship between a bending force and a magnitude of displacement ulnar deviation of the wrist.

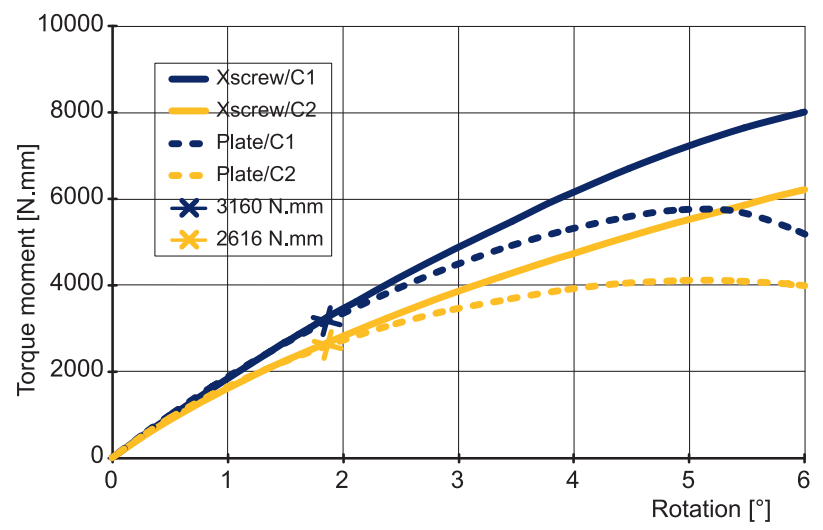

Fig. 7. Relationship between a torque moment and rotation in forearm pronation.

engineering. It makes calculations less demanding and removes the risk of false stress values on the rims of threads. As proven, this simplification makes no difference on the stability of the system as a whole and its stiffness or the strength of each of its components.

The geometric model of a radius was constructed from a series of CT images of a healthy subject's wrist (resolution, $512 \times 512$ pixels; single pixel size, $0.412 \mathrm{~mm}$; section distance, $0.5 \mathrm{~mm}$ ) in a *.DICOM format regularly used for saving CT images of individual sections. In a specialized Mimics 12 software (Materialise, Leuven, Belgium), these served to create a 3D geometric model of the distal radius and its triangular surface mesh in an *.inp format. Using ABAQUS 6.9 software, 3D finite element grid was subsequently constructed.

The ABAQUS 6.9 programme was used for all calculations in this research. Both fragments of the radius bone and components of osteosynthesis implants were meshed by linear, four-sided solid C3D4 elements; only the models of $\mathrm{K}$-wires were meshed by linear, six-sided solid C3D8 elements.

\section{Material models}

Ti6A14V titanium alloy are used for both implants, was simulated as a homogenous, isotropic and elastoplast material in all analyses performed.

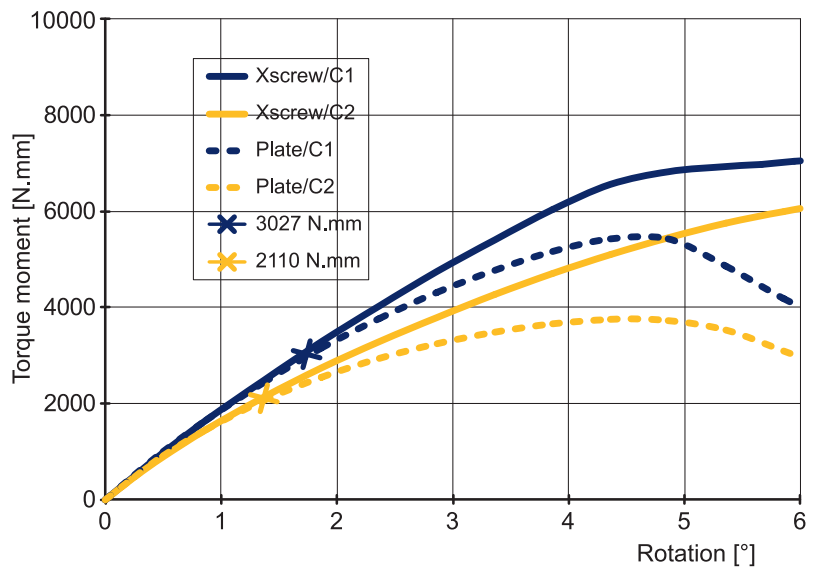

Fig. 8. Relationship between a torque moment and rotation in forearm supination.

Bone tissue in all analyses was simulated as a non-homogenous, isotropic and elastoplast material. For each bone fragment, mechanical properties were determined by the width of compact bone and trabecula bone density in the corresponding area of the distal radius on the healthy patient's CT scan. The density of the bone was distinguished by grades of grey; with lighter colour indicating a higher bone density.

Mathematical modelling also presented the bone tissue as a material when the ultimate failure load was exceeded. Responded by a reduced stiffness and load-bearing capacity, in certain circumstances this can be interpreted as "bone tissue disturbance". The ultimate failure load results in permanent damage to the material. If load exceeds yield stress, the material is irreversibly degraded. These can be illustrated with the example of a steel wire. If loaded with a small force, the wire will bend. If the force stops acting, the wire will resume its original shape. If the wire is loaded in excess of yield point, it will remain bent forever but will still keep together. However, if loading force is still higher and even exceeds breaking strength, the wire will break into two parts.

Damage to bone tissue in this case is not understood as a complete loss of integrity resulting in a fracture, but as a local defect inside or on the surface of a bone. This is the sample of stripping the thread of a screw in the bone. The bone as a whole is undamaged and retains its stiffness although, in the near vicinity of the screw, the bone tissue is permanently damaged. As a result, the screw is no longer firmly anchored in the position.

\section{Boundary conditions and loading}

The analyses were performed to determine stability of the whole complex, i.e., fractured bone fixed with an osteosynthetic implant, subjected to loading forces. In all analyses, the proximal part of the radius was firmly fixed in space. Therefore, any action of external forces was unable to move in any direction. For calculations, the model was gradually subjected to forced loads simulating tension (in the long bone axis), bending (volar and dorsal flexion; radial and ulnar deviation) and rotation (pronation and supination).

In the models of fractures fixed with angle-stable plates, the connection of a plate with screws and connection of bone fragments 
$107-111$

with screws were designated together as a TIE connection. To "tie" can mean to link, bind, join, lock or knot and, figuratively, the TIE connection makes the two surfaces placed one over the other behave as if they were "glued" or "knotted". In ABAQUS, this specific type of joining includes two contact parts whose knots are firmly tied together and the connection transfers a displacement from the superior to the inferior contact surface without changing its value. Therefore, it is able to transfer both tension and compression. In simple terms, this connection can be regarded as a "glued joint" of two parts.

For contacts existing among fragments of the radius, among components of the intramedullary X-screw system and between the implant and bone, a normal "HARD" contact, with a coefficient of friction $(f)=0.15$, was simulated. The designation HARD indicates that two contact surfaces hit each other hard during displacement. This type of contact involves two options: the surfaces can lean against each other or can detach themselves. In comparison with the TIE connection, only pressing force can be transferred. This model simulates the real situation where incorporation of parts is not possible, nevertheless their detachment is permissible.

\section{Results}

All computer simulation models were created as contact, nonlinear and static tasks aimed at finding responses (or stability) of the whole system, i.e., an implant-fixed fracture, to a load applied. The finite element modelling took in great consideration, in a great detail, the mechanical properties of bone tissue at the site of osteosynthetic. For the comparison of the stability of osteosynthesis using the angle-stable plate Medin with that of intramedullary X-screw system, the model was gradually loaded with a forced pre-defined displacement or rotation until any part of the model system failed. This allowed us to evaluate the behaviour of a fractured bone fixed with an implant after its loading by forces much exceeding those acting at normal activities or involved in mechanisms of injury. Attention was paid not to the evaluation of tension of individual components, but to the stability of the whole system until its complete failure. Displacement of the reference knot located in the centre of the articular surface of the wrist joint is presented in Figures 2 to 8. Displacement of individual bone fragments can't be seen in the graphs. A sudden sharp change in curve shape indicates a failure resulting from an irreversible collapse of any part of the system due to a rapid fall in loading forces, with a displacement still increasing.

Regarding the axial compressive load of the radius from Figure 3 , it is apparent that intramedullary osteosynthesis remained stable and stiff until the maximum simulated load. On the other hand, plate osteosynthesis began to lose stability at a compressive load of $164 \mathrm{~N}$ in type $\mathrm{C} 1$ and at $141 \mathrm{~N}$ in type $\mathrm{C} 2$ fractures. A load higher than these values resulted in a complete failure (Fig. 3).

\section{Axial compressive load of the radius}

From Figure 2 it is apparent that intramedullary osteosynthesis remained stable and stiff until the maximum simulated load. On the other hand, plate osteosynthesis began to lose stability at a compressive load of $164 \mathrm{~N}$ in type $\mathrm{C} 1$ and at $141 \mathrm{~N}$ in type $\mathrm{C} 2$ fractures. A load higher than these values resulted in complete failure.

\section{Bending loads}

Volar flexion. In plate osteosynthesis, a complete loss of stability ensued under volar bending forces of $204 \mathrm{~N}$ and $113 \mathrm{~N}$ applied to type $\mathrm{C} 1$ and type $\mathrm{C} 2$ fractures, respectively. Intramedullary osteosynthesis, on the other hand, remained stable under these loads in both fracture types (Fig. 3).

Dorsal flexion. Stability of the whole model system in dorsal flexion was almost identical for both osteosynthetic techniques in the two fracture types studied, only with plate osteosynthesis being slightly stiffer (by about $1 \%$ ) (Fig. 4).

Radial deviation. Plate osteosynthesis lost stability under a load of 7089 N.mm in type C1 fracture and under 7501 N.mm in type $\mathrm{C} 2$ fracture. Intramedullary osteosynthesis remained stable at these values in both fractures (Fig. 5).

Ulnar deviation. Loading in ulnar deviation resulted in the loss of stability of intramedullary osteosynthesis at a force of 2874 N.mm and 2100 N.mm in type $\mathrm{C} 1$ and $\mathrm{C} 2$ fractures, respectively, while plate osteosynthesis was able to withstand these forces and thus proved to remain stiffer under ulnar deviation loads (Fig. 6).

\section{Axial torsional load of the forearm}

Pronation. In type $\mathrm{C} 1$ facture, a torque moment of 3138 N.mm produced a rapid decrease in plate osteosynthesis stability in comparison with intramedullary fixation. The type $\mathrm{C} 2$ fracture responded similarly. Value of torque for plate osteosynthesis was limited to 2509 N.mm. Differences in stiffness between the two fixation methods increased with increasing loads in both fracture types (Fig. 7).

Supination. Both implants behaved as in forearm pronation, only a rapid loss of stability in plate osteosynthesis was recorded at lower values, i.e., a torque moment of 3027 N.mm for type C1 fracture and that of 2130 N.mm for type C2 fracture. Also, as in pronation, differences in stiffness between the implants increased with increased loads in both fracture types (Fig. 8).

\section{Discussion}

Using the mathematical modelling, the stability of type $\mathrm{C} 1$ and $\mathrm{C} 2$ fractures of the distal radius fixed by either angle-stable plate osteosynthesis or intramedullary X-screws was evaluated with loading in various hand and arm positions, i.e., volar and dorsal flexion, radial and ulnar deviation, forearm pronation and supination and with axial loading of the radius. The implant stability was tested for all types of wrist motion that can be used in daily postoperative activities and as part of rehabilitation programmes. For completeness, these loads should have included an axial tensile load applied to the radius as an antagonist load of compression. However, since these loads are never used in physical therapy, there was no reason to include it in the mathematical modelling.

The mathematical model showed that in dorsal flexion, the stability of intramedullary osteosynthesis was comparable with that of plate osteosynthesis. Plate osteosynthesis proved to be more stable only in ulnar deviation. On application of loads in volar flexion, radial deviation, pronation and supination, and in axial loading of the radius, intramedullary osteosynthesis provided more stability to type $\mathrm{C} 1$ and $\mathrm{C} 2$ fractures than plate osteosynthesis. 
It should be kept in mind that the mathematical modelling was performed with certain simplifications and generalizations. For the models constructed as static, only one loading moment and a response of the system was described. In reality, fractures treated with either method are post-operatively subjected to repeated low intensity loading, and this may be one of the causes of gradual implant loosening and change in alignment.

Another factor affecting the interpretation of our results is the mode of loading. In the model, forces from the reference knot were distributed over the distal radial articular surface. In real situations, muscles attached to fragments of the distal radius can greatly modify the effect of loading on the articular surface.

Unfortunately, this limitation of the mathematical modelling used will exist until relevant information on the strength of individual muscles involved in wrist joint movements is published. In addition, daily activities combine all three types of loading, i.e., compression, bending and rotation. This fact was not considered in our mathematical modelling.

Despite the reservations mentioned above, our results can be regarded as a helpful contribution to the use of plate versus intramedullary osteosynthesis for distal radius fractures as the aim of the study was to compare these implants under identical conditions.

Good stability of locking plate osteosynthesis in distal radius fractures has been reported in several biomechanical studies (8, 9 ). Its superiority over osteosynthesis without a locked screw plate interface was proved (10-12). Evidence was also provided that in type A3 fractures, volar fixed-angle plates with threaded locking screws were mechanically superior to volar fixed-angle plates with smooth locking pegs under torsional loading (13).

So far only a few studies comparing the stability of locking plate and intramedullary osteosyntheses have been published. A recent experimental study demonstrated that an intramedullary fixation of a distal AO-A3 radial fracture is biomechanically more stable than volar fixed-angle plating (14). Angle-stable plate and intramedullary (Micronail) osteosyntheses provided a comparable stability for distal radius fractures (15). An identical or higher stability has been demonstrated for the intramedullary screw Targon DR, as compared with three volar locking plates under volar and dorsal bending loads (16).

Our study concluded that the results of our mathematical modelling were in concurrence with those of other authors. However, for technical reasons, the use of intramedullary osteosynthesis for distal radius fractures was limited in comminuted intra-articular fractures in which the size of a displaced radial styloid fragment is not large enough. Therefore, the placement of an intramedullary system itself resulted in loss of fragment alignment. On the other hand, osteosynthetic plates adjusted in shape to the bone surface help to achieve anatomical reduction of fragments.

\section{Conclusion}

The conclusions drawn from the mathematical modelling comparing the plate and intramedullary osteosynthesis hold equally for both type $\mathrm{C} 1$ and type $\mathrm{C} 2$ fractures of the distal radius. The intramedullary Xscrew system is stiffer and more stable because it can withstand higher loads than plate fixation, with no loss of stability and without reduction of stiffness; under loads of less than $15 \mathrm{~kg}$ the stability of both systems is comparable.

After waging all these fact and making the best choice for clinical medicine, it is necessary to consider differences in plate and intramedullary screwing techniques because of their different potentials for maintenance of anatomical reduction.

\section{References}

1. Fitoussi F, Ip WY, Chow SP. Treatment of displaced intra-articular fractures of the distal end of the radius with plates. J Bone Joint Surg Am 1997; 79: 1303-1312.

2. Gruber G, Gruber K, Giessauf C, Clar H, Zacherl M, Fuerst F, Bernhardt GA. Volar plate fixation of AO type $\mathrm{C} 2$ and $\mathrm{C} 3$ distal radius fractures, a single-center study of 55 patients. J Orthop Trauma 2008; 22: 467-472.

3. Murakami K, Abe Y, Takahashi K. Surgical treatment of unstable distal radius fractures with volar locking plates. J Orthop Sci 2007; 12: 134-140.

4. Osada D, Kamei S, Masuzaki K, Takai M, Kameda M, Tamai K. Prospective study of distal radius fractures treated with a volar locking plate system. J Hand Surg Am 2008; 33 691-700.

5. van Vugt R, Geerts RW, Werre AJ. Osteosynthesis of distal radius fractures with the Micronail. Eur J Trauma Emerg Surg 2010; 6: 471-476.

6. Gradl G, Senft M, Gierer P, Beck M, Mittlmeier T. Fixation of distal radial fractures with the Targon DR nail. Oper Orthop Traumatol 2009; 21: 472-483.

7. Harreld K, Li Z. Intramedullary fixation of distal radius fractures. Hand Clin 2010; 26: 363-372.

8. Chen L, Dai Q, Wongworawat MD. A biomechanical comparison between two volar locking plate systems for distal radius fractures. Orthopedics 2006; 29: 927-929.

9. Liporace FA, Kubiak EN, Neony GK, Iesaka K, Egol KA, Koval KJ. A biomechanical comparison of two volar locked plates in a dorsally unstable distal radius fracture model. J Trauma 2006; 61: 668-762.

10. Levin SM, Nelson CO, Botts JD, Teplitz GA, Kwon Y, Serra-Hsu F. Biomechanical evaluation of volar locking plates for distal radius fractures. Hand (NY) 2008; 3: 55-60.

11. Rudig L, Mehling I., Klitscher D, Mehler D, Prommersberger KJ, Rommens PM, Müller LP. Biomechanical study of four palmar locking plates and one non-locking palmar plate for distal radius fractures: stiffness and load to failure tests in a cadaver model. Biomed Tech (Berl) 2009; 54: 150-158.

12. Sobky K, Baldini T, Thomas K, Bach J, Williams A, Wolf JM. Biomechanical comparison of different volar fracture fixation plates for distal radius fractures. Hand (NY) 2008; 3: 96-101.

13. Weninger P, Dall'Ara E, Leixnering M, Pezzei C, Hertz H, Drobet H, Redl H, Zysset P. Volar fixed-angle plating of extra-articular distal radius fractures - a biomechanical analysis comparing threaded screws and smooth pegs. J Trauma 2010; 69: 46-55.

14. Burkhart KJ, Nowak TE, Gradl G, Klitscher D. Mehling I, Mehler D, Müller LP, Rommens PM. Intramedullary nailing vs. palmar locked plating for unstable dorsally comminuted distal radius fractures: a biomechanical study. Clin Biomech (Bristol, Avon) 2010; 25: 771-775.

15. Capo JT, Kinchelow T, Brooks K, Tan V, Manigrasso M, Francisco K. Biomechanical stability of four fixation constructs for distal radius fractures. Hand (NY) 2009; 4: 272-278.

16. Konstantinidis L, Helwig P, Seifert J, Hirschmüller A, Liodakis E, Südkamp NP, Oberst M. Internal fixation of dorsally comminuted fractures of the distal part of the radius: a biomechanical analysis of volar plate and intramedullary nail fracture stability. Arch Orthop Trauma Surg 2011; 131: 1529-1537.

Received March 19, 2012. Accepted October 27, 2013. 